\title{
Robot Vision
}




\title{
Sensor Planning Techniques and Active Visual Inspection
}

\author{
Vito Roberto and Emanuele Trucco \\ Machine Vision Laboratory, \\ Department of Mathematics and Informatics \\ University of Udine, Italy \\ Department of Computing and Electrical Engineering \\ Heriot-Watt University, Edinburgh, Scotland
}

\begin{abstract}
This paper addresses the problem of computing the sequence of positions in space (inspection script) from which a robot-mounted sensor can perform a given inspection task optimally. We present the design and partial realisation of a sensor planning system, GASP, capable of generating inspection scripts for a variety of tasks, objects and sensors. The treatment of detection uncertainty is discussed with reference to the problem of detecting line features; the generalised sensor is introduced, and novel results in a simulated environment are reported.
\end{abstract}

\section{Introduction. Sensor Planning and Inspection}

A key feature of active vision systems is the ability to reconfigure themselves in order to improve their performance at given visual tasks. This ability can be exploited in advanced inspection, for instance by directing robot-mounted sensors to positions in space from which an inspection task can be performed optimally, e.g. the features to inspect are maximally visible, or most robustly detected by the vision system, or both. In general, several parts (features) of an object need inspecting, and each one is best observed from a different viewpoint. Therefore, a sequence of sensor positioning actions (an inspection script) must be planned to perform the task $[5,6,7]$. Visual inspection strategies and their automatic generation depend on the type and number of sensors and object features to be inspected: therefore, models of both sensors and objects must be available.

Typical inspection tasks that a planner can afford are the following. Single-feature, single-sensor: find the position from which a single imaging sensor (e.g., intensity or range) can inspect a single object features optimally. Singlefeature, multiple-sensor: find the position from which a multiple sensor (e.g., a stereo head) can inspect a feature optimally. Multiple-feature, single-sensor: (a) find the position from which a sensor can simultaneously inspect a set of features optimally; (b) find the best path for a sensor to inspect a set of features from optimal positions. Multiple-feature, multiple-sensor: find the best path taking a stereo head to inspect a set of features from optimal positions.

This paper is a brief overview of GASP (General Automatic Sensor Planning), a system capable of generating inspection scripts for a variety of tasks, 
objects and sensors. The features currently examined by GASP are surface patches (planes, cylinders, cones, delimited by their possible intersections) and segments (straight lines and curves defined by the intersections of patches, as above). Using a feature-based CAD model of the object to be inspected, GASP identifies the viewpoints from which each feature is visible, how much, and how reliably it can be detected. We present the inspection tasks currently considered by GASP (section 2); section 3 concerns the treatment of uncertainty based on the concept of generaised sensor and its novel results.

\section{Inspection Scripts}

We have designed a representation, the FIR - Feature Inspection Representation, used by GASP to plan inspection scripts and carry them out in a simulated environment.

The (discrete) set of all the viewpoints accessible to the sensor is called visibility space. A FIR partitions the visibility space into visibility regions, each formed by all the viewpoints from which a given feature is visible. Viewpoints are weighted by two coefficients, visibility and reliability. The former indicates the size of the feature in the image (the larger the image, the larger the coefficient); the latter expresses the expected robustness with which the feature can be detected. The two coefficients are linearly combined into an optimality coefficient, in such a way that the relative importance of visibility and reliability can be adjusted.

In GASP, the visibility space is modelled by a geodesic dome [3] centered on the object; the viewpoints are the centers of the dome's facets. The algorithm to compute the FIR [7] generates a geodesic dome around a CAD model of the object to inspect, then raytraces the model from each viewpoint on the dome. A FIR for the widget shown in Figure 1 (left), observed from 320 viewpoints and with image resolution of $64 \times 64$ pixels, occupies about $80 \mathrm{Kbytes}$. The widget is about $250 \mathrm{~mm}$ in length.

\subsection{Single-sensor Scripts}

Single-feature Inspection. In a FIR, the viewpoints belonging to a visibility region are arranged in a list ordered by optimality. Figure 1 (right) shows the visibility region of the planar patch in Figure 1 (left) given a single intensity camera of focal length about $50 \mathrm{~mm}$; the widget has been magnified for clarity. The object-camera distance (dome radius) was determined by GASP as the minimum one such that the widget is visible from all viewpoints; other choices are possible. Viewpoints have been shaded according to their optimality (the darker the better), assuming equal weights for visibility and reliability.

Simultaneous Inspection of Several Features. In this case, a sensor must inspect several features simultaneously. The region of the visibility space from which a set of features is visible is called covisibility region, and is obtained as the intersection of the visibility regions of the individual features. This is done 

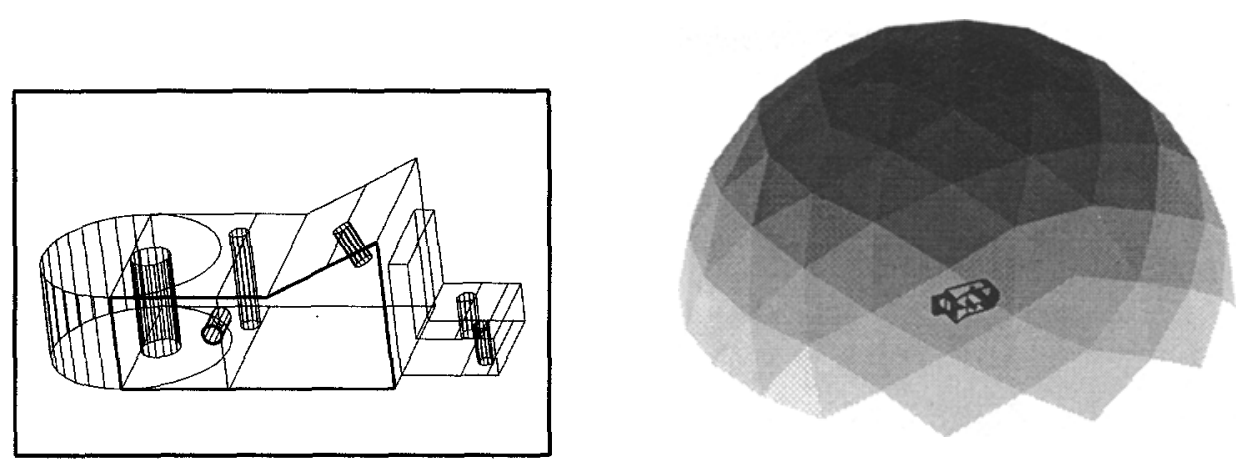

Figure 1: CAD model of a widget (left) and visibility region for highlighted planar patch (right).

simply and efficiently thanks to the FIR's structure. Notice that the merit (optimality) of a viewpoint for inspecting the set of features must be defined as a function of the optimality of the viewpoint for inspecting each individual feature.

Sequential Inspection of Several Features. Given a set of features, we now want to find a path in space which takes the sensor through the optimal inspection positions associated to all the features in the set; the path must also be as short as possible since we wish to minimise the number of sensor repositioning actions. Planning the shortest 3-D path through a given set of points is a NP-complete problem - the Travelling Salesman Problem, TSP - and only approximate solutions can be found. Three TSP algorithms have been designed and tested [8]: simulated annealing, CCAO [4], and the elastic net [2]. We found that, with up to 100 sites, CCAO outperformes the other two in terms of path length and distance from the overall optimal solution. An example of inspection path is given in Figure 2 (right) in the context of multiple-sensor, multiple-feature scripts.

\subsection{Multiple-sensor Scripts}

Single-feature Inspection. We want to determine the position from which a stereo head can observe at best a given feature. Using a FIR and information about the head geometry, GASP selects efficiently the most promising positions of the head inside the region, and picks the best one. Notice that the optimality must be defined as a combination of those of the (distinct!) viewpoints in which the two cameras are placed [8]. Figure 2 (left) shows the optimal placement for a stereo head found by GASP in the large (compared with the head's camerato-camera distance) visibility region of one of the widget's back planar surfaces. Multiple-feature Inspection. This class of scripts finds the shortest path for a 

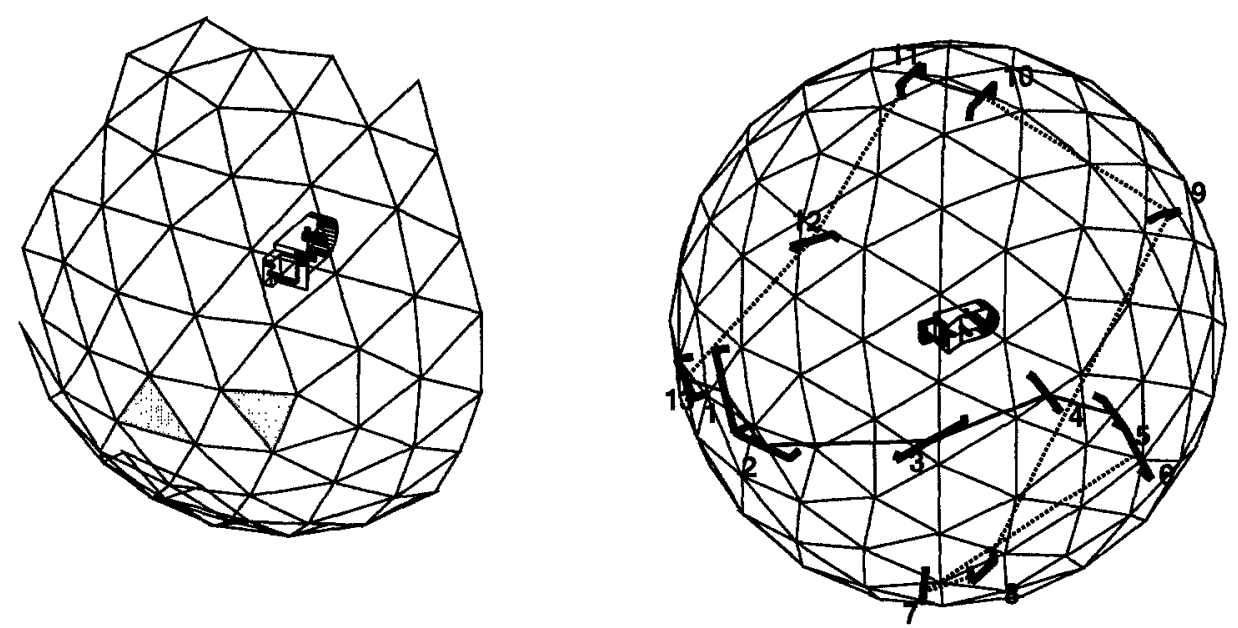

Figure 2: Stereo head inspection (left) and inspection path (right).

stereo head. Thanks to the uniform representation of viewpoint optimality in the FIR (i.e., referred to the viewpoint, not to the sensor), it is possible to apply the same path-finding module used for the single-sensor case. An example of optimal shortest 3-D inspection path is shown in Figure 2 (right). It has been computed assuming two intensity cameras of focal length about $50 \mathrm{~mm}$ and image resolution $64 \times 64$. The dome radius (object-camera distance) was fixed by GASP at $2400 \mathrm{~mm}$. The camera-to-camera distance is $500 \mathrm{~mm}$. 13 surfaces of the widget are visible to the cameras (others are too small or mostly occluded, e.g. the bottom surfaces of holes); the 13 viewpoints are the sites visited by the stereo head on the shortest path computed.

\section{Modelling Sensor Reliability}

A FIR records how much of a feature is visible from a viewpoint, and how reliably the feature can be detected by a given module. Reliability coefficients refer therefore to specific feature extractors - e.g., a line detector, a module computing the length of a linear feature, and so forth. Since the whole complexity of sensing and measuring is embedded in the two FIR coefficients - visibility and reliability - we call generalised sensor the compound formed by the physical sensor and the measuring or detection module.

The reliability has been estimated by simulation. To explain this we shall refer to features detected by an intensity camera and a line extractor. In this case our generalised sensor comprised a pinhole camera (perspective, no distortion), a Canny edge extractor, and a Hough line detector. We generated synthetic images of linear features across a wide range of the relevant parameters: line length and strength, noise variance. The images were corrupted with additive Gaussian noise, of variance in a range centeredon the values estimated from real images acquired by a real camera. For each image, we identified and located 


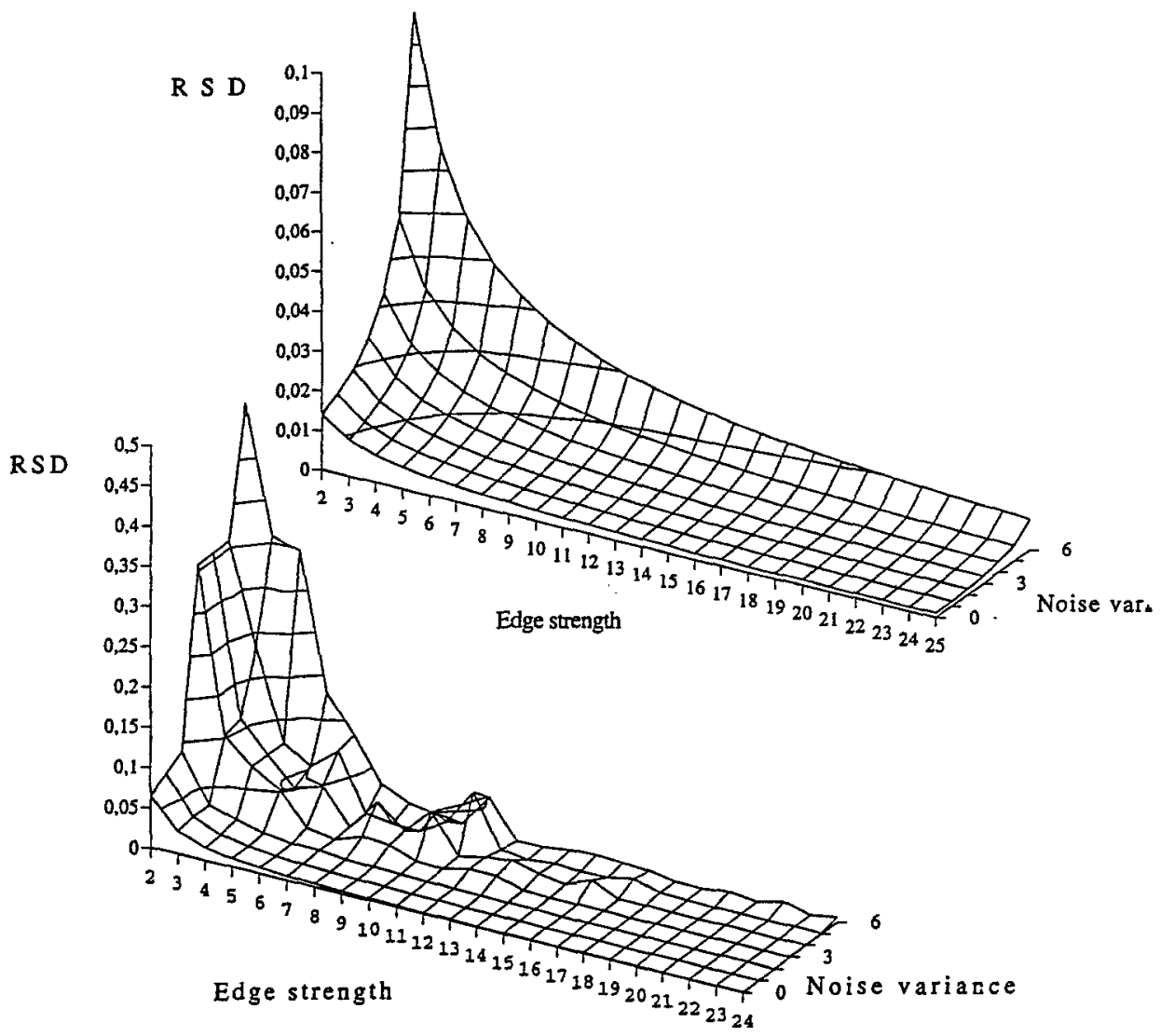

Figure 3: Uncertainty estimates via Root Square Distance (RSD). Experimental data (lower left) and interpolated values (upper right).

line using the target edge and line detectors. We then defined the uncertainty for the whole linear feature as a weighted sum of the observed variances of three measures: the positional uncertainty of each line point [1], the root square distance of each edge point from the estimated line (with subpixel accuracy) and the strength of each edge point. Second-order uncertainty surfaces interpolating the experimental values express the uncertainty of the detection for all possible occurrences of the line in an image. As an example, Figure 3 shows the measured uncertainties for the root square distance (left) and the corresponding interpolated surface (right), as functions of edge strength and noise.

The latter surfaces are used when building a FIR to enter appropriate reliability values for each feature and viewpoint. Since a FIR is generated by raytracing $C A D$ models, the values of all parameters necessary to index the surface are always known, and a reliability coefficient is associated to each pair viewpoint-feature simply as the complement to one of uncertainty. At the moment, reliability data are available for linear features extracted from intensity images and surface patches extracted from range images. 


\section{Conclusions}

Planning the positions from which a mobile sensor can perform visual inspection tasks optimally is an important capability of flexible robotic systems. We have briefly presented the main functionalities of GASP, a sensor planning system which generates inspection scripts for various tasks, objects and sensors. Examples have been shown of the performances of GASP in a simulated environment. Uncertainty estimates have been discussed, based on the concept of generalised sensor; the latter takes into account the uncertainties arising from both the sensing and the measuring tools. Experimental data have been reported. Current and future work on GASP include incorporating more and more realistic sensor and workspace models, as well as running GASP-generated scripts in a real inspection setup.

\section{References}

1. J. Canny: A computational approach to edge detection. IEEE Transactions on Pattern Analysis and Machine Intelligence 6, 679-698 (1986).

2. R. Durbin, D. Willshaw: an analogue approach to the travelling salesman problem. Nature 326, April, 689-691 (1987).

3. G. Fekete, L.S. Davis: Property spheres: a new representation for 3-D object recognition. In: Proceedings IEEE Workshop on Computer Vision, Representation and Control, (IEEE, Inc.), 192-201 (1984).

4. B.L. Golden, W.R. Stewart: Empirical Analysis and Heuristics. In E.L. Lawler, J.K. Lenstra, A.H.G. Rinnooy Kan, and D.B. Shmoys (eds.), The Traveling Salesman Problem, Wiley Interscience Pubs. 1985, Cap. 7, pp.227-239.

5. K.D. Gremban, K. Ikeuchi: Planning multiple observations for object recognition. International Journal of Computer Vision 12(2/3), 137-172 (1994).

6. K. Tarabanis, R.Y. Tsai, P.K. Allen: Analytical characterization of feature detectability constraints. Computer Vision, Graphics and Image Processing: Image Understanding 59(3), 340-358 (1994).

7. E. Trucco, E. Thirion, M. Umasuthan, A.M. Wallace: Visibility scripts for active feature-based inspection. In Proceedings British Machine Vision Conference, Springer-Verlag 1991, 538-547.

8. E. Trucco, M. Diprima, V. Roberto: Visibility scripts for active feature-based inspection. Pattern Recognition Letters 15, 1151-1164 (1994). 